\title{
Concise Research Reports
}

\section{Acetaminophen or Tylenol? A Retrospective Analysis of Medication Digital Communication Practices}

\author{
David Ouyang, $M D^{7}$, Rebecca Tisdale, $M D^{2}$, Euan Ashley, $M B B S, P h D^{7}$, Jeffrey $C h i, M D^{2}$, and \\ Jonathan H. Chen, MD, PhD ${ }^{2}$
}

'Department of Internal Medicine, Division of Cardiovascular Medicine, Stanford University School of Medicine, Stanford, CA, USA; ${ }^{2}$ Department of Internal Medicine, Division of Hospital Medicine, Stanford University School of Medicine, Stanford, CA, USA.

KEY WORDS: Pharmacoeconomics; physician sentiment; provider communication; choosing wisely; hidden curriculum.

J Gen Intern Med 33(8):1218-20

DOI: $10.1007 / \mathrm{s} 11606-018-4455-1$

(c) Society of General Internal Medicine 2018

\section{BACKGROUND}

Prescribing of brand name drugs when generic alternatives exist has been linked to decreased patient adherence and worse health outcomes. ${ }^{1}$ Furthermore, failure to substitute brand name for generic drugs results in billions of dollars in estimated excess costs to individual patients and the US health system yearly. ${ }^{2}$ Using drugs' trade names rather than generic names in clinical practice has been shown to increase usage of these brand name drugs over their generic equivalents, even when pharmacy substitution is available. ${ }^{3}$ American medical schools have therefore included policies supporting use of nonproprietary drug names in the clinical setting as part of a range of interventions to promote generic prescribing and limit industry influence on medical students and trainees. ${ }^{4}$

To what extent physicians and other healthcare providers continue to use trade names versus generic names in clinical communication is difficult to measure; however, text pages represent a contemporaneous record of communication across members of the multidisciplinary healthcare team, including pharmacists and nursing staff, and can be used to assess fluency with medication naming.

\section{OBJECTIVE}

Using a novel large-scale approach, we sought to characterize the relative use of trade and generic drug names in the inpatient setting.

\section{METHODS AND FINDINGS}

We analyzed 1,048,576 text pages to internal medicine residents staffing inpatient medicine services between June 1, 2013 and April 24, 2017 at a large academic university hospital. Mentions of medications by both generic and brand names were identified and tallied. When explicitly mentioned in the body of the text

Published online May 1, 2018 page, the occupation of the sender was established. Multivariate analysis was performed on the sender occupation, number of syllables, consonants, and vowels in both the generic and trade names. Text pages were algorithmically processed using Python and statistical tests were performed in R. This study was approved by the Stanford University IRB.

We identified 102,243 pages regarding medications, of which $34,489(33.7 \%)$ pages were from nurses and 20,446 (20.0\%) pages were from pharmacists (Fig. 1). Sixty-two medications (i.e., unique active ingredients) were mentioned more than 240 times. The most common classes of medications were analgesics (12.9\%), antibiotics (12.9\%), and anticoagulants (11.3\%). There was significant variation in the preferential use of brand names versus generic names (Fig. 1). Nurses were more likely than pharmacists to use trade names (OR 39.9, 95\% CI 26.5-53.3, $p=0.004)$. A greater number of syllables in generic name compared to the trade name was also correlated with trade name usage (OR 3.1, 95\% CI 1.8-4.5, $p=0.022$ ). There was no interaction between profession and number of syllables.

\section{DISCUSSION}

Text paging behaviors reflect real-world, informal provider-toprovider communication. By applying a high-throughput method of assessing medication naming sentiment, we show that there is significant variability in preference of brand name versus generic name usage for common drugs in the inpatient setting. For certain drugs, the brand name was used overwhelmingly more than the generic - in some cases, by nearly 100 to 1 . For example, nurses preferentially mentioned Haldol and Zofran rather than haloperidol and ondansetron. These observations suggest that the propagation of brand names in this academic medical center comes from many avenues, including from non-physician members of the multidisciplinary treatment team. We also found a preference for medication names with fewer syllables, although this effect may be confounded by the space-limited context of text pages.

Though our analysis draws on the depth of over one million text pages, its limitation to one institution may constrain generalizability. Our institution has similar trainee demographics to the general American medical school population; however, the surrounding patient population generally represents a wealthier and more ethnically diverse population. ${ }^{5}$ 


NPH
Albuterol
Metoprolol
Ceftriaxone
Tramadol
Warfarin
Lispro
Amlodipine
Oxycodone
Levofloxacin
Docusate
Carvedilol
Quetiapine
Glargine
Pantoprazole
Gabapentin
Enoxaparin
Calcium Carbonate
Prochlorperazine
Lorazepam
Zolpidem
Metoclopramide
Furosemide
Acetaminophen
Hydrocodone
Hyodromorphone
Pipercillin-Tazobactam
Haloperidol
Ondansetron

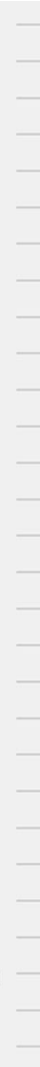

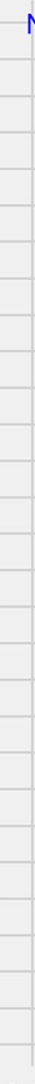

0.01

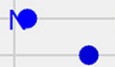

- iP
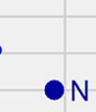

$P$
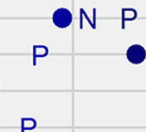

$P$

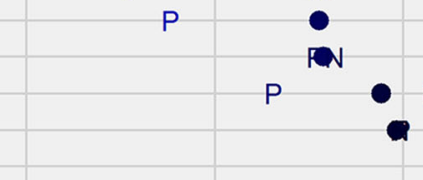

$N$

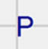

$P{ }_{N}^{N}$

$P$

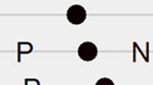

$P$

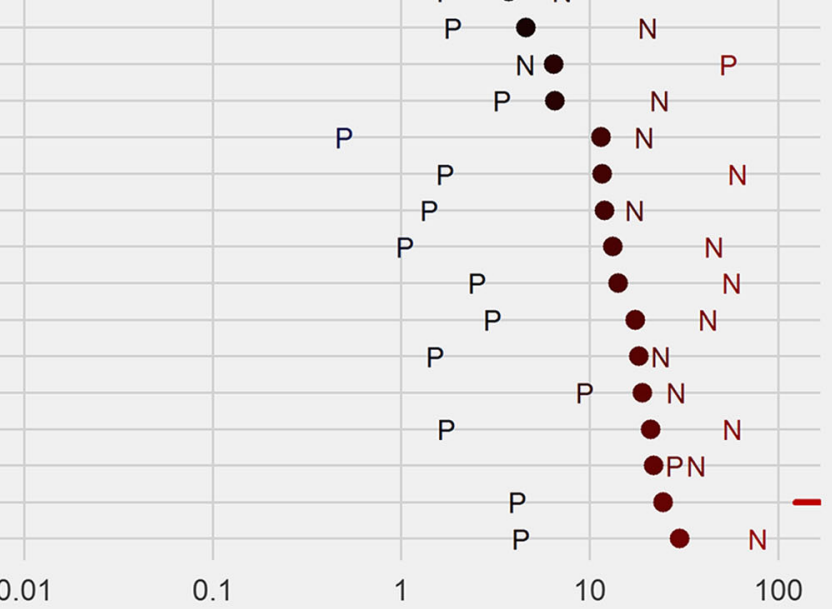

Ratio of Generic Name to Brand Name

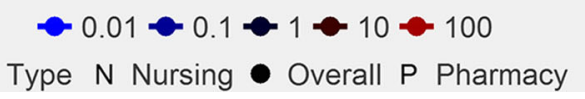

Humulin/Novolin

Proair/Proventil

Toprol/Lopressor

Rocephin

Ultram

Coumadin

Humalog

Norvasc

Percocet/Roxicodone

Levaquin

Colace

Coreg

Seroquel

Lantus

Protonix

Neurontin

Lovenox

TUMS

Compazine

Ativan

Ambien

Reglan

Lasix

Tylenol

Norco/Vicodin

Dilaudid

Zosyn

Haldol

Zofran

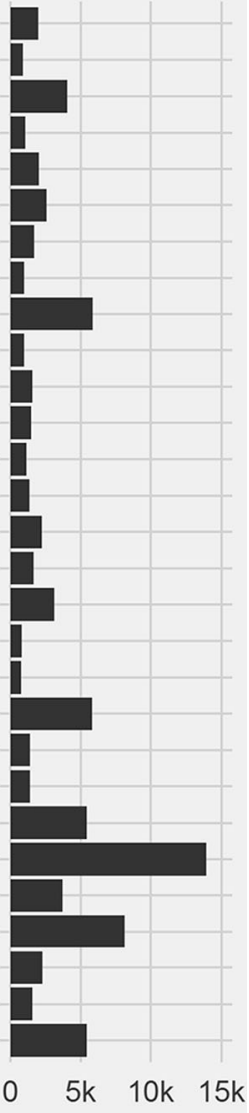

Number of Pages

Fig. 1 Incidence and ratio of medication mentions by brand name and generic name.

Internal medicine societies have identified a need to develop strategies for increasing the relative use of generic drugs over their brand-name counterparts. ${ }^{6}$ Structural changes like adopting electronic medical record systems that standardize medication names, limiting industry influence, increasing provider awareness of generic medication names may improve the relative use of generic drugs. Insofar as our findings reflect variable community standards for medication name usage across different health professions, educational interventions to reduce brand name use within specific communities may be effective in furthering this effort. Further work should be done to assess the impact of educational and quality improvement initiatives to reduce brand name use and the impact on prescribing behaviors.

Acknowledgements: The authors would like to thank Dr. Paul Cheng and Dr. Jody Shen for constructive criticism of the manuscript.

Corresponding Author: David Ouyang, MD; Department of Internal Medicine, Division of Cardiovascular Medicine Stanford University School of Medicine, Stanford, CA, USA (e-mail: ouyangd@stanford. edu).
Author contributions Dr. Ouyang had full access to all of the data in the study and takes responsibility for the integrity of the data and the accuracy of the data analysis.

Study concept and design: Ouyang.

Acquisition of data: Ouyang, Chi, and Chen.

Analysis and interpretation of data: Ouyang, Tisdale, Ashley, Chi, and Chen.

Drafting of the manuscript: Ouyang and Tisdale.

Critical revision of the manuscript for important intellectual content: Ouyang, Tisdale, Ashley, Chi, and Chen.

Statistical analysis: Ouyang, Tisdale, and Chen.

\section{Compliance with Ethical Standards:}

Conflict of Interest: The authors declare that they do not have a conflict of interest.

\section{REFERENCES}

1. Gagne JJ, Choudhry NK, Kesselheim AS, Polinski JM, Hutchins D, Matlin OS, Brennan TA, Avorn J, Shrank WH. Comparative effectiveness of generic and brand-name statins on patient outcomes: a cohort study. Ann Intern Med 2014;161(6):400-407. doi: https://doi. org/10.7326/M13-2942

2. Kale MS, Bishop TF, Federman AD, Keyhani S. "Top 5" lists top \$5 billion (Research Letter). Arch Intern Med 2011;171(20):1856-1858. doi: https:// doi.org/10.1001/archinternmed.2011.501. 
3. Mott DA, Cline RR. Exploring generic drug use behavior: the role of prescribers and pharmacists in the opportunity for generic drug use and generic substitution. Med Care 2002;40(8):662-674. doi:https://doi.org/ 10.1097/01.MLR.0000020926.85284.8E.

4. Obley AJ, Iossi KA, Humphrey LL. Teaching Wisely. J Grad Med Educ 2014;6(3):587-588. doi:https://doi.org/10.4300/JGME-D-14-00335.1.

5. AAMC. U.S. Medical School Applications and Matriculants by School, State of Legal Residence, and Sex, 2017-2018. Available: https://www. aamc.org/data/facts/applicantmatriculant/85986/byinstitution.html
6. Choudhry NK, Denberg TD, Qaseem A. Improving adherence to therapy and clinical outcomes while containing costs: opportunities from the greater use of generic medications: best practice advice from the Clinical Guidelines Committee of the American College of Physicians. Ann Intern Med 2016;164:41-49. https://doi.org/10. 7326/M14-2427 